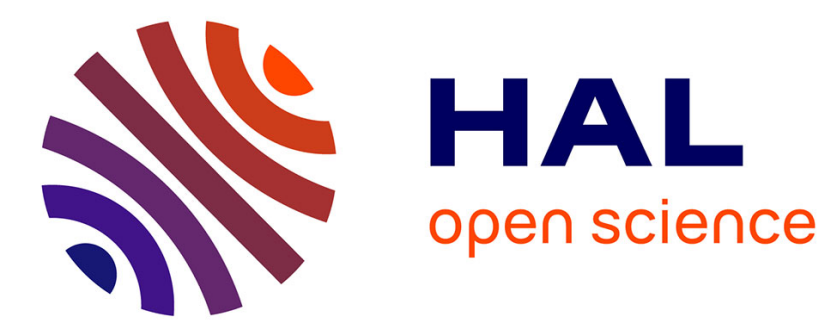

\title{
Properties of Tight Frames that are Regular Schemes
}

\author{
Malcolm Egan
}

\section{To cite this version:}

Malcolm Egan. Properties of Tight Frames that are Regular Schemes. Cryptography and Communications - Discrete Structures, Boolean Functions and Sequences , 2020, 12, pp.499-510. 10.1007/s12095019-00378-2 . hal-02148974

\section{HAL Id: hal-02148974 https://hal.inria.fr/hal-02148974}

Submitted on 6 Jun 2019

HAL is a multi-disciplinary open access archive for the deposit and dissemination of scientific research documents, whether they are published or not. The documents may come from teaching and research institutions in France or abroad, or from public or private research centers.
L'archive ouverte pluridisciplinaire HAL, est destinée au dépôt et à la diffusion de documents scientifiques de niveau recherche, publiés ou non, émanant des établissements d'enseignement et de recherche français ou étrangers, des laboratoires publics ou privés. 


\title{
Properties of Tight Frames that are Regular Schemes
}

\author{
Malcolm Egan
}

Received: date / Accepted: date

\begin{abstract}
Finite frames are sequences of vectors in finite dimensional Hilbert spaces that play a key role in signal processing and coding theory. In this paper, we study the class of tight unit-norm frames for $\mathbb{C}^{d}$ that also form regular schemes, which we call tight regular schemes (TRS). Many common frames that arise in applications such as equiangular tight frames and mutually unbiased bases fall in this class. We investigate characteristic properties of TRSs and prove that for many constructions, they are intimately connected to weighted 1-designs - arising from cubature rules for integrals over spheres in $\mathbb{C}^{d}$ - with weights dependent on the Voronoi regions of each frame element. Aided by additional numerical evidence, we conjecture that all TRSs in fact satisfy this property.
\end{abstract}

Keywords tight frames $\cdot$ regular schemes $\cdot$ spherical designs

\section{Introduction}

Finite frames play an important role in signal processing and coding theory as a means of providing redundant representations of elements in finite dimensional complex Hilbert spaces. For example, frames form the basis for the construction of good measurement matrices in compressed sensing [4], codebooks for vector quantization [12], and coding for erasures [22]. Connections have also been established to measurement operators in quantum information theory [20].

Despite the range of applications there are a few special classes of frames are ubiquitous, including equiangular tight frames (ETFs) [22, 28, 8] and mutually

Malcolm Egan

CITI Laboratory

Univ. Lyon, INSA-Lyon, INRIA

Lyon, France

E-mail: malcom.egan@inria.fr 
unbiased bases (MUBs) [27]. A key feature of these frames is that they are tight unit-norm frames with a low coherence; that is, the maximum squared magnitude of inner products, or angles, between distinct frame elements is small. Low coherence is a particularly important property for reconstruction of signals from a sparse approximation [4].

Construction of tight frames with a low coherence has proven to be a difficult challenge. One approach to tackle this problem is to restrict the number of distinct angles presented by the frame. In [6], constructions of real valued tight frames with at most $k$ angles were derived. In [23], unitary representations of cyclic and dihedral groups were exploited to obtain tight frames with few angles and low coherence.

In this paper, we study finite unit-norm tight frames that are regular schemes, called tight regular schemes (TRSs). This class of frames includes ETFs and MUBs as special cases [17,18], as well as a wide range of frames constructed using unitary representations of finite groups. The defining feature of frames that are regular schemes is the presence of the property that the set of angles from any frame element obtained by taking the squared magnitude of inner products with all other frame elements is the same. Due to the restricted number of distinct angles, this provides a basis to control the coherence.

We investigate special cases of TRSs for different size angle sets. A key observation is that for angle sets of size one and two, ETFs and MUBs, respectively, naturally arise. We also demonstrate that the existence of TRSs with a full angle set depends on whether or not the size of the frame is even or odd. We observe that certain subsets of unitary representations of finite groups yield TRSs; namely, the group covariant set. As a consequence, TRSs include a large class of unit-norm tight frames. We also show that such TRSs have Voronoi regions with equal area.

Although the coherence describes one aspect of the geometry of a frame, the Voronoi regions capture properties of the geometry beyond pairwise relationships between frame elements. As group-based TRSs have Voronoi regions with equal area, this implies that these frames form weighted 1-designs [21] with weights given by the areas of the Voronoi regions. A natural question is then whether other TRSs have the same property. We show that a finite frame obtained via Alltop's quadric polyphase construction [1] also forms a weighted 1-design with weights given by the areas of the Voronoi regions, which are not all equal. Numerical experiments also suggest that this property also holds for MUBs obtained from Alltop's quadric and cubic polyphase constructions [1] as well as ETFs obtained from difference sets [28] and Steiner systems [13]. Based on this theoretical and numerical evidence, we conjecture that in fact all TRSs form weighted 1-designs with weights determined by Voronoi region areas. 


\section{Preliminaries}

\subsection{Tight Frames}

Before formally defining TRSs, we recall the notions of a tight frame and a regular scheme. A family of vectors $\left(\boldsymbol{\varphi}_{i}\right)_{i=1}^{M}$ in $\mathbb{C}^{d}$ is called a finite frame for $\mathbb{C}^{d}$ if there exist constants $0<A \leq B<\infty$ such that

$$
A\|\mathbf{x}\|^{2} \leq \sum_{i=1}^{M}\left|\mathbf{x}^{\dagger} \boldsymbol{\varphi}_{i}\right|^{2} \leq B\|\mathbf{x}\|^{2}, \forall \mathbf{x} \in \mathbb{C}^{d},
$$

where $(\cdot)^{\dagger}$ denotes the conjugate transpose. If $\left\|\boldsymbol{\varphi}_{i}\right\|=1, i=1,2, \ldots, M$, then $\left(\boldsymbol{\varphi}_{i}\right)_{i=1}^{M}$ is called a unit norm frame. Clearly, any finite sequence of unit-norm vectors forms a finite frame for $\mathbb{C}^{d}$ as long as the vectors span $\mathbb{C}^{d}$.

An important class of frames arises when $A=B$ in which the family of vectors $\left(\boldsymbol{\varphi}_{i}\right)_{i=1}^{M}$ is called a tight frame [4] [25]. The initial importance of tight frames is due to their role as a generalization of an orthonormal basis. In particular if $\left(\boldsymbol{\varphi}_{i}\right)_{i=1}^{M}$ is a tight frame, then for any $\mathbf{x} \in \mathbb{C}^{d}$,

$$
\mathbf{x}=\frac{1}{A} \sum_{i=1}^{M}\left(\mathbf{x}^{\dagger} \boldsymbol{\varphi}_{i}\right) \boldsymbol{\varphi}_{i}
$$

An important characterization of tight frames is via the frame potential [2].

Definition 1 If $\left(\boldsymbol{\varphi}_{i}\right)_{i=1}^{M}$ in $\mathbb{C}^{d}$ is a unit-norm frame, the frame potential of $\left(\boldsymbol{\varphi}_{i}\right)_{i=1}^{M}$ is given by

$$
\operatorname{FP}\left(\left(\boldsymbol{\varphi}_{i}\right)_{i=1}^{M}\right)=\sum_{i, j=1}^{M}\left|\boldsymbol{\varphi}_{i}^{\dagger} \boldsymbol{\varphi}_{j}\right|^{2}
$$

Theorem 1 [2] The unit-norm frame $\left(\boldsymbol{\varphi}_{i}\right)_{i=1}^{M}$ in $\mathbb{C}^{d}$ is tight if and only if the frame potential is minimized; that is,

$$
\operatorname{FP}\left(\left(\boldsymbol{\varphi}_{i}\right)_{i=1}^{M}\right)=\frac{M^{2}}{d} .
$$

The coherence of a unit-norm frame $\left(\boldsymbol{\varphi}_{i}\right)_{i=1}^{M}$ in $\mathbb{C}^{d}$ is defined as

$$
\max _{i \neq j}\left|\boldsymbol{\varphi}_{i}^{\dagger} \boldsymbol{\varphi}_{j}\right|^{2}
$$

By a theorem of Welch [26], the coherence is lower bounded by

$$
\max _{i \neq j}\left|\boldsymbol{\varphi}_{i}^{\dagger} \boldsymbol{\varphi}_{j}\right|^{2} \geq \frac{M-d}{d(M-1)},
$$

known as the Welch bound. 
In this paper, we are concerned with the class of tight frames known as regular schemes [18]. These frames are defined by their angle set, which for the frame $\left(\varphi_{i}\right)_{i=1}^{M}$ in $\mathbb{C}^{d}$ is

$$
\mathcal{A}=\left\{\left|\boldsymbol{\varphi}_{j}^{\dagger} \boldsymbol{\varphi}_{k}\right|^{2}\right\}_{1 \leq j<k \leq M}
$$

and define

$$
\mathcal{A}_{j}=\left\{\left|\boldsymbol{\varphi}_{j}^{\dagger} \boldsymbol{\varphi}_{k}\right|^{2}\right\}_{k \neq j} .
$$

Associated to each frame element $\varphi_{j}$ and angle $\alpha \in \mathcal{A}$ is the sub-degree of $\left(\boldsymbol{\varphi}_{i}\right)_{i=1}^{M}$, which is defined as

$$
d_{\alpha}(j)=\left|\left\{1 \leq k \leq M: k \neq j,\left|\boldsymbol{\varphi}_{j}^{\dagger} \boldsymbol{\varphi}_{k}\right|^{2}=\alpha\right\}\right| .
$$

Definition 2 Let $\left(\boldsymbol{\varphi}_{i}\right)_{i=1}^{M}$ be a unit norm frame in $\mathbb{C}^{d}$ with angle set $\mathcal{A}$. If for each $\alpha \in \mathcal{A}$, the sub-degree $d_{\alpha}(i)$ is independent of $i$, then $\left(\boldsymbol{\varphi}_{i}\right)_{i=1}^{M}$ is called a regular scheme. If a regular scheme $\left(\boldsymbol{\varphi}_{i}\right)_{i=1}^{M}$ also forms a tight frame, then $\left(\varphi_{i}\right)_{i=1}^{M}$ is said to be a tight regular scheme (TRS).

A useful alternative characterization of TRSs is given in the following proposition, which follows immediately from the definition.

Proposition 1 Let $\left(\boldsymbol{\varphi}_{i}\right)_{i=1}^{M}$ in $\mathbb{C}^{d}$ be a tight unit norm frame. Then, $\left(\boldsymbol{\varphi}_{i}\right)_{i=1}^{M}$ is a TRS if and only if the sets $\mathcal{A}_{j}, j=1, \ldots, M$ are identical.

\subsection{Regular Schemes and $t$-Designs}

There is a long-known connection between regular schemes and spherical $t$ designs (henceforth called $t$-designs) [17]. Following [18], let $\operatorname{Hom}(k, l)$ be the subset of the polynomial ring $\mathbb{C}\left[x_{1}, \ldots, x_{d}, y_{1}, \ldots, y_{d}\right]$ that consists of all polynomials that are homogeneous of degree $k$ in the variables $x_{1}, \ldots, x_{d}$ and homogeneous of degree $l$ in the variables $y_{1}, \ldots, y_{d}$. To each polynomial $p$ in $\operatorname{Hom}(k, l)$ associate a function $p_{\circ}$ on the sphere $S^{d-1}$ by defining $p_{\circ}(\zeta)=p\left(\zeta, \zeta^{*}\right)$ for $\zeta \in S^{d-1}$. Define $\operatorname{Hom}(k, k)_{\circ}=\left\{p_{\circ}: p \in \operatorname{Hom}(k, k)\right\}$.

Definition 3 Let $\mu$ be the unique normalized $\mathcal{U}(d)$-invariant Haar measure on $\mathbb{C} S^{d-1}$. A finite non-empty subset $X$ of $\mathbb{C} S^{d-1}$ is a $t$-design in $\mathbb{C} S^{d-1}$ if and only if the cubature formula

$$
\frac{1}{|X|} \sum_{x \in X} f(x)=\frac{1}{\mu\left(\mathbb{C} S^{d-1}\right)} \int_{\mathbb{C} S^{d-1}} f(x) d \mu(x)
$$

holds for all $f \in \operatorname{Hom}(t, t)_{\circ}$.

Intuitively, a $t$-design provides a means of expressing the expectation of homogeneous polynomials in terms of an average over a finite set on points. The following theorem due to Hoggar [17] provides a link between regular schemes and $t$-designs. 
Theorem 2 [17, Theorem 2.4] Let $\left(\boldsymbol{\varphi}_{i}\right)_{i=1}^{M}$ in $\mathbb{C}^{d}$ be a regular scheme with angle set $\mathcal{A}$. Then, $\left(\boldsymbol{\varphi}_{i}\right)_{i=1}^{M}$ is a t-design if and only if

$$
1+\alpha_{1}^{r} d_{1}+\cdots+\alpha_{s}^{r} d_{s}=M \frac{(1)_{r}}{(d)_{r}}, r=0,1, \ldots, t
$$

where $(a)_{r}=a(a+1) \cdots(a+r-1)$.

Since 1-designs are equivalent to tight frames, Theorem 2 shows that TRSs are a non-trivial class of regular schemes. We now turn to classes of tight regular schemes constrained by the size of their angle sets.

\section{Examples of Tight Regular Schemes}

$3.1|\mathcal{A}|=1$

Consider the case that $|\mathcal{A}|=1$. We first observe that in this case, TRSs are intimately linked to ETFs which have a coherence achieving equality in the Welch bound.

Definition 4 Let $\left(\boldsymbol{\varphi}_{i}\right)_{i=1}^{M}$ be a unit-norm frame in $\mathbb{C}^{d}$. Then, $\left(\boldsymbol{\varphi}_{i}\right)_{i=1}^{M}$ is an equiangular tight frame (ETF) if and only if

$$
\max _{j \neq i}\left|\boldsymbol{\varphi}_{i}^{\dagger} \boldsymbol{\varphi}_{j}\right|^{2}=\frac{M-d}{d(M-1)} .
$$

That is, $\left(\boldsymbol{\varphi}_{i}\right)_{i=1}^{M}$ satisfies the Welch bound [22].

Proposition 2 Suppose that $\left(\boldsymbol{\varphi}_{i}\right)_{i=1}^{M}$ in $\mathbb{C}^{d}$ is a TRS with $\mathcal{A}=\{\alpha\}$. Then, $\left(\boldsymbol{\varphi}_{i}\right)_{i=1}^{M}$ achieves equality in the Welch bound. That is, $\left(\boldsymbol{\varphi}_{i}\right)_{i=1}^{M}$ is an equiangular tight frame.

Proof It follows from the definition of $\left(\boldsymbol{\varphi}_{i}\right)_{i=1}^{M}$ and Theorem 1 that

$$
1+(M-1) \alpha=\frac{M}{d}
$$

Hence,

$$
\alpha=\frac{M-d}{d(M-1)}
$$

as required. 
$3.2|\mathcal{A}|=2$

As in the case $|\mathcal{A}|=1$, frames with extremal angle sets also arise for $|\mathcal{A}|=2$. To formalize this claim, first recall the Levenshtein bound [19].

Theorem 3 (Levenshtein) Let $\left(\boldsymbol{\varphi}_{i}\right)_{i=1}^{M}$ in $\mathbb{C}^{d}$ be a unit norm finite frame. Then,

$$
\max _{1 \leq i<j \leq M}\left|\boldsymbol{\varphi}_{i}^{\dagger} \boldsymbol{\varphi}_{j}\right|^{2} \geq \frac{2 M-d^{2}-d}{(d+1)(M-d)} .
$$

The following result characterizes TRSs that achieve the Levenshtein bound.

Proposition 3 Suppose that $\left(\boldsymbol{\varphi}_{i}\right)_{i=1}^{M}$ in $\mathbb{C}^{d}$ is a TRS with $\mathcal{A}=\{0, \alpha\}$. Then, $\left(\boldsymbol{\varphi}_{i}\right)_{i=1}^{M}$ forms a complex projective 2-design if and only if $\left(\boldsymbol{\varphi}_{i}\right)_{i=1}^{M}$ achieves equality in the Levenshtein bound.

Proof Since $\left(\boldsymbol{\varphi}_{i}\right)_{i=1}^{M}$ is a complex projective 2-design by [17, Theorem 2.4],[18, Theorem 1] if and only if

$$
\frac{1}{M}\left(1+d_{\alpha} \alpha^{2}\right)=\frac{2}{d(d+1)} .
$$

and

$$
\sum_{j=1}^{M}\left|\boldsymbol{\varphi}_{i}^{\dagger} \boldsymbol{\varphi}_{j}\right|^{2}=1+\alpha d_{\alpha}=\frac{M}{d}, i=1, \ldots, M
$$

which implies that $d_{\alpha}=\frac{M-d}{d \alpha}$. It then follows that

$$
\frac{1}{M}\left(1+\frac{M-d}{d} \alpha\right)=\frac{2}{d(d+1)} .
$$

Solving for $\alpha$ then yields

$$
\alpha=\frac{2 M-d^{2}-d}{(d+1)(M-d)}
$$

which is precisely the condition for equality in the Levenshtein bound.

We remark that this result can also be extracted from [14, Proposition 3.3]. Moreover, complete sets of mutually unbiased bases form complex projective 2-designs with angle set $\left\{0, \frac{1}{d}\right\}$. Such examples have been widely studied, with numerous constructions $[3,29,10,30,15,9]$.

As also noted in [14], further constructions of frames that achieve the Levenshtein bound can be obtained from Hoggar's work on regular schemes that also form complex projective 2-designs. From [17, Example 7], there is a TRS in $\mathbb{C}^{6}$ with $M=126$ and $\mathcal{A}=\left\{0, \frac{1}{4}\right\}$, obtained from the diameters of a complex polytope. 
Example 1 The frame consisting of the vectors in $\mathbb{C}^{6}$, given by

$$
\frac{1}{\sqrt{2}}\left(\begin{array}{c}
1 \\
-\omega^{k_{0}} \\
0 \\
0 \\
0 \\
0
\end{array}\right), \frac{1}{\sqrt{6}}\left(\begin{array}{c}
1 \\
\omega^{k_{1}} \\
\omega^{k_{2}} \\
\omega^{k_{3}} \\
\omega^{k_{4}} \\
\omega^{k_{5}}
\end{array}\right),
$$

and all coordinate permutations, where $\omega=e^{2 \pi i / 3}, k_{i}=0,1,2$ and $k_{1}+\cdots k_{5}=$ $0(\bmod 3)$.

Example 1 is remarkable in that no other finite frame meeting the Levenshtein bound is known in $\mathbb{C}^{6}$ with size exceeding 36 . In particular, it is conjectured that a complete set of mutually unbiased bases does not exist in this dimension [11].

Another example of a finite frame achieving the Levenshtein bound is the complete set of mutually unbiased bases in $\mathbb{C}^{4}$, leading to a frame with $M=20$ elements. Using the complex projective 2-design in $\mathbb{C}^{4}[17$, Example 6], leads to a finite frame in $\mathbb{C}^{4}$ achieving the Leveneshtein bound with $M=40$ elements and angle set $\mathcal{A}=\left\{0, \frac{1}{3}\right\}$.

Example 2 The frame consisting of the vectors in $\mathbb{C}^{4}$, given by

$$
\begin{aligned}
& \left(\begin{array}{l}
1 \\
0 \\
0 \\
0
\end{array}\right),\left(\begin{array}{l}
0 \\
1 \\
0 \\
0
\end{array}\right),\left(\begin{array}{l}
0 \\
0 \\
1 \\
0
\end{array}\right),\left(\begin{array}{l}
0 \\
0 \\
0 \\
1
\end{array}\right), \frac{1}{\sqrt{3}}\left(\begin{array}{c}
0 \\
1 \\
-\omega_{1} \\
\omega_{2}
\end{array}\right), \\
& \frac{1}{\sqrt{3}}\left(\begin{array}{c}
-\omega_{1} \\
0 \\
1 \\
\omega_{2}
\end{array}\right), \frac{1}{\sqrt{3}}\left(\begin{array}{c}
\omega_{1} \\
-\omega_{2} \\
0 \\
1
\end{array}\right), \frac{1}{\sqrt{3}}\left(\begin{array}{c}
1 \\
\omega_{1} \\
\omega_{2} \\
0
\end{array}\right),
\end{aligned}
$$

where $\omega_{1}, \omega_{2}$ run independently through cube roots of 1 .

The next example was first introduced as a complex projective 2-design in [7] and detailed in [16, Example 18]. It is a finite frame in $\mathbb{C}^{5}$ with $M=45$ elements and angle set $\mathcal{A}=\left\{0, \frac{1}{4}\right\}$.

Example 3 The frame consisting of vectors in $\mathbb{C}^{5}$, given by

$$
\left(\begin{array}{l}
1 \\
0 \\
0 \\
0 \\
0
\end{array}\right), \frac{1}{\sqrt{4}}\left(\begin{array}{c}
0 \\
1 \\
\pm \omega \\
\pm \omega \\
1
\end{array}\right)
$$

under all cyclic permutations with $\omega=e^{2 \pi i / 3}$. 
$3.32<|\mathcal{A}|<M-1$

For $|\mathcal{A}|>2$, there appears to be no known variational characterization of TRSs analogous to the Welch or Levenshtein bounds. Nevertheless, Theorem 2 and the constructions in [17] provide examples of TRSs angle sets in this regime. As an illustration, [16, Example 12] yields a TRS in $\mathbb{C}^{3}$ with 21 elements and angle set $\left\{0, \frac{1}{4}, \frac{1}{2}\right\}$.

$3.4|\mathcal{A}|=M-1$

We now consider TRSs with angle sets $|\mathcal{A}|=M-1$. The following example demonstrates that it is not always possible to construct such a TRS $\left(\boldsymbol{\varphi}_{i}\right)_{i=1}^{M}$ in $\mathbb{C}^{d}$ for all $M$.

Example 4 Let $M=3$ and $d=2$ and suppose that $\left(\boldsymbol{\varphi}_{i}\right)_{i=1}^{M}$ in $\mathbb{C}^{2}$ has Gram matrix $\mathbf{G}$ with entries corresponding to inner products between each frame element. Define the matrix $\mathbf{M}=\mathbf{G} \circ \mathbf{G}^{*}\left(\mathbf{G}^{*}\right.$ is the entry-wise conjugate of $\mathbf{G})$, and consider the completion problem

$$
\mathbf{M}=\left(\begin{array}{ccc}
a_{1} & a_{2} & a_{3} \\
a_{2} & a_{1} & ? \\
a_{3} & ? & a_{1}
\end{array}\right) .
$$

This completion problem does not have a solution such that $\mathbf{M}$ is symmetric as there is either a violation of the requirement that each column should have distinct elements or that each row should have distinct elements. As such, the $\operatorname{TRS}\left(\boldsymbol{\varphi}_{i}\right)_{i=1}^{M}$ with $|\mathcal{A}|=2$ does not exist.

In fact, TRSs with $|\mathcal{A}|=M-1$ do not exist for any odd $M>1$. This is proven in the following theorem, which relies on a connection to the existence problem of symmetric Latin squares.

Definition 5 Let $\{1, \ldots, M\}$ be the alphabet. An order $M$ Latin square $\mathbf{L}$ is a $M \times M$ matrix constructed such that each row and column contains each element of the alphabet only once. The Latin square $\mathbf{L}$ is symmetric if $\mathbf{L}=\mathbf{L}^{T}$.

Theorem 4 Let $a_{1}=1$ and $a_{2}, \ldots, a_{M}$ be the elements of the angle set $\mathcal{A}$ of the TRS $\left(\boldsymbol{\varphi}_{i}\right)_{i=1}^{M}$ in $\mathbb{C}^{d}$. If $a_{1}, \ldots, a_{M}$ are distinct. Then, $M$ is even.

Proof Let $\mathbf{G}$ be the Gram matrix of $\left(\boldsymbol{\varphi}_{i}\right)_{i=1}^{M}$ and $\mathbf{M}=\mathbf{G} \circ \mathbf{G}^{*}\left(\mathbf{G}^{*}\right.$ is the entry-wise conjugate of $\mathbf{G})$. Suppose that the elements of $\mathbf{M}$ are mapped to the elements of a matrix $\mathbf{L}$ via $a_{i} \mapsto i$. Under the assumption that each $a_{i}$ is distinct, $\mathbf{L}$ is a Latin square with alphabet $\{1, \ldots, M\}$. Suppose that only the diagonal of $\mathbf{L}$ is specified, then it is not always possible to complete the Latin square and hence guarantee that $\left(\boldsymbol{\varphi}_{i}\right)_{i=1}^{M}$ is a TRS with $|\mathcal{A}|=M-1$. In fact by [5, Section 3], for the completion of a prescribed diagonal to a symmetric $M \times M$ Latin square to exist, it is necessary and sufficient that the diagonal 
contains each element exactly once for odd $M$ and an even number of times for even $M$. Since $a_{1}$ appears $M$ times on the diagonal of $\mathbf{L}$, it follows that $M$ must be even.

It is straightforward to construct TRSs that have $M-1$ distinct angles when $M$ is even. We present an example below.

Example 5 An example of a TRS $\left(\boldsymbol{\varphi}_{i}\right)_{i=1}^{4}$ in $\mathbb{C}^{2}$ with $M-1$ distinct elements in its angle set can be obtained as follows. Define the unitary matrices

$$
\begin{aligned}
& \mathbf{M}_{1}=\left(\begin{array}{ll}
1 & 0 \\
0 & 1
\end{array}\right), \mathbf{M}_{2}=\left(\begin{array}{cc}
0 & -1 \\
1 & 0
\end{array}\right), \mathbf{M}_{3}=\left(\begin{array}{cc}
1 & 0 \\
0 & -1
\end{array}\right) \\
& \mathbf{M}_{4}=\left(\begin{array}{cc}
0 & -1 \\
-1 & 0
\end{array}\right)
\end{aligned}
$$

and the vector

$$
\phi=\frac{1}{\sqrt{0.3^{2}+0.7^{2}}}\left(\begin{array}{l}
0.3 \\
0.7
\end{array}\right)
$$

Then, the frame with elements

$$
\varphi_{i}=\mathbf{M}_{i} \phi
$$

can be readily shown to be tight and hence is a TRS with angle set of the form $\mathcal{A}=\left\{0, \alpha_{1}, \alpha_{2}\right\}, \alpha_{1}, \alpha_{2} \in(0,1)$.

In fact, the construction in Example 5 exploits the group structure of the matrices $\left\{M_{i}\right\}$. We further develop the connection between TRSs and groups in the following section.

\section{Group Covariant Tight Regular Schemes}

We now consider a class of TRSs that are obtained from unitary representations of finite groups.

Definition 6 A finite group $G \subset \mathcal{U}\left(\mathbb{C}^{d}\right)$ is irreducible if, for every $\phi \neq 0, \phi \in$ $\mathbb{C}^{d}$

$$
\operatorname{span}(G \phi)=\mathbb{C}^{d}
$$

where $G \boldsymbol{\phi}$ is the $G$-orbit of $\boldsymbol{\phi}$ defined by the set $\{\mathbf{g} \phi: \mathbf{g} \in G\}$.

Lemma 1 Let $G \subset \mathcal{U}(d)$ be an irreducible finite group. Then, the G-orbit of every unit-norm $\phi \in \mathbb{C}^{d}$ is a TRS $\left(\boldsymbol{\varphi}_{i}\right)_{i=1}^{M}$ in $\mathbb{C}^{d}$. 
Proof By [24, Theorem 6.3], $\left(\boldsymbol{\varphi}_{i}\right)_{i=1}^{M}$ is tight. All that remains is to show that each angle set is the same. Viewing each angle as distinct, observe that for all $i=1, \ldots, M$

$$
\begin{aligned}
\left\{\left|\boldsymbol{\varphi}_{i}^{\dagger} \boldsymbol{\varphi}_{j}\right|^{2}: j \in\{1,2, \ldots, M\}\right\} & =\left\{\left|\boldsymbol{\varphi}_{1}^{\dagger} \mathbf{U}_{i}^{\dagger} \mathbf{U}_{j} \boldsymbol{\varphi}_{1}\right|^{2}: j \in\{1,2, \ldots, M\}\right\} \\
& =\left\{\left|\boldsymbol{\varphi}_{1}^{\dagger} \mathbf{U}_{k} \boldsymbol{\varphi}_{1}\right|^{2}: k \in\{1,2, \ldots, M\}\right\}
\end{aligned}
$$

where we used the fact that $\mathbf{U}_{i} \mathbf{U}_{j} \in G$ for all $i, j \in\{1,2, \ldots, M\}$.

Definition 7 Let $\left\{\mathbf{U}_{i}\right\}_{i=1}^{M}$ be a set of unitary matrices in $\mathcal{U}(d)$. The set $\left\{\mathbf{U}_{i}\right\}_{i=1}^{M}$ is group covariant if for all $j, k=1, \ldots, M, \mathbf{U}_{j} \mathbf{U}_{k}=e^{i \theta_{j k}} \mathbf{U}_{l}$ for some $\theta_{j k} \in[0,2 \pi)$ and $\mathbf{U}_{l} \in\left\{\mathbf{U}_{i}\right\}_{i=1}^{M}$.

Proposition 4 Let $G$ be a group, $\phi \in \mathbb{C}^{d}$ be unit-norm and $H$ be the set of group covariant matrices obtained from $G$. If $G \phi$ is a tight frame, then $H \boldsymbol{\phi}$ is a TRS.

Proof We first establish tightness. Since $G \boldsymbol{\phi}$ is tight, it follows that

$$
\sum_{\mathbf{U} \in G} \mathbf{U} \phi \phi^{\dagger} \mathbf{U}^{\dagger}=\frac{|G|}{d} \mathbf{I} .
$$

Equivalently,

$$
\sum_{\mathbf{U} \in H}|I(d)| \mathbf{U} \phi \phi^{\dagger} \mathbf{U}^{\dagger}=\frac{|G|}{d} \mathbf{I}
$$

where $|I(d)|$ is the size of the normal subgroup of $G$ arising from the equivalence relation $\mathbf{g} \sim e^{i \theta} \mathbf{g}$. As a consequence,

$$
\sum_{\mathbf{U} \in H} \mathbf{U} \phi \phi^{\dagger} \mathbf{U}^{\dagger}=\frac{|H|}{d} \mathbf{I},
$$

since $|H||I(d)|=|G|$ by Lagrange's theorem. Hence $H \boldsymbol{\phi}$ is tight.

Now, $H \phi$ is group covariant and hence, viewing each angle as distinct, for any $\mathbf{U}_{i} \in H$,

$$
\left\{\left|\boldsymbol{\phi}^{\dagger} \mathbf{U}^{\dagger} \mathbf{U}_{i} \boldsymbol{\phi}\right|^{2}\right\}_{\mathbf{U} \in H}=\left\{\left|\boldsymbol{\phi}^{\dagger} e^{i \theta} \mathbf{U} \boldsymbol{\phi}\right|^{2}\right\}_{\mathbf{U} \in H}
$$

This implies that $H \boldsymbol{\phi}$ is also a regular scheme, completing the proof. 


\section{Tight Regular Schemes and Weighted 1-Designs}

A feature of any TRS $\left(\boldsymbol{\varphi}_{i}\right)_{i=1}^{M}$ in $\mathbb{C}^{d}$ constructed from a set of group-covariant unitary matrices is that the area of the Voronoi regions corresponding to the elements $\varphi_{i}$ are equal. Let $S^{d-1}$ be the unit sphere in $\mathbb{C}^{d}$. The Voronoi region corresponding to $\boldsymbol{\varphi}_{i}$ is then defined by

$$
\left\{\mathbf{z} \in S^{d-1}:\left|\mathbf{z}^{\dagger} \boldsymbol{\varphi}_{i}\right|^{2} \geq\left|\mathbf{z}^{\dagger} \boldsymbol{\varphi}_{j}\right|^{2}, j \neq i\right\} .
$$

The area of the Voronoi region corresponding to $\boldsymbol{\varphi}_{i}$ is given by

$$
V_{i}=\mu\left(\left\{\mathbf{z} \in S^{d-1}:\left|\mathbf{z}^{\dagger} \boldsymbol{\varphi}_{i}\right|^{2} \geq\left|\mathbf{z}^{\dagger} \boldsymbol{\varphi}_{j}\right|^{2}, j \neq i\right\}\right),
$$

where $\mu$ is the unique normalized $\mathcal{U}(d)$-invariant Haar measure. We then have the following result.

Theorem 5 Let $\left\{\mathbf{U}_{i}\right\}_{i=1}^{M}$ be a group covariant set of $d \times d$ unitary matrices and $\phi \in \mathbb{C}^{d}$ a unit norm vector. If $\left(\boldsymbol{\varphi}_{i}\right)_{i=1}^{M}$ in $\mathbb{C}^{d}$ is a frame with elements $\left(\mathbf{U}_{i} \phi\right)_{i=1}^{M}$, then $V_{i}=\frac{1}{M}$.

Proof Observe that

$$
\begin{aligned}
V_{i} & =\mu\left(\left\{\mathbf{z} \in S^{d-1}:\left|\mathbf{z}^{\dagger} \boldsymbol{\varphi}_{i}\right|^{2} \geq\left|\mathbf{z}^{\dagger} \boldsymbol{\varphi}_{j}\right|^{2}, j \neq i\right\}\right) \\
& =\mu\left(\left\{\mathbf{z}^{\prime}=\mathbf{U}_{i}^{\dagger} \mathbf{z} \in S^{d-1}:\left|\mathbf{z}^{\prime \dagger} \mathbf{U}_{i}^{\dagger} \boldsymbol{\varphi}_{i}\right|^{2} \geq\left|\mathbf{z}^{\prime \dagger} \boldsymbol{\varphi}_{j}\right|^{2}, j \neq i\right\}\right) \\
& =V_{1} .
\end{aligned}
$$

Since $\mu$ is a normalized measure, the result follows.

Since the frame $\left(\boldsymbol{\varphi}_{i}\right)_{i=1}^{M}$ is tight, by Theorem 1 it follows that

$$
\sum_{i, j=1}^{M} V_{i} V_{j}\left|\boldsymbol{\varphi}_{i}^{\dagger} \boldsymbol{\varphi}_{j}\right|^{2}=\frac{1}{d} .
$$

As a consequence, TRSs constructed from sets of group covariant unitary matrices are closely related to a variation of $t$-designs, defined as follows.

Definition 8 Let $\left(\boldsymbol{\varphi}_{i}\right)_{i=1}^{M}$ in $\mathbb{C}^{d}$ be a unit norm frame and $\left(w_{i}\right)_{i=1}^{M}$ be weights satisfying $\sum_{i=1}^{M} w_{i}=1$. Then, $\left(\boldsymbol{\varphi}_{i}\right)_{i=1}^{M}$ is a weighted 1-design with weights $\left(w_{i}\right)_{i=1}^{M}$ if for all $1 \leq k \leq t$

$$
\sum_{i, j=1}^{M} w_{i} w_{j}\left|\boldsymbol{\varphi}_{i}^{\dagger} \boldsymbol{\varphi}_{j}\right|^{2}=\frac{1}{d} .
$$

In light of Definition $8,\left(\boldsymbol{\varphi}_{i}\right)_{i=1}^{M}$ forms a weighted 1-design with weights $\left.\left(V_{i}\right)\right)_{i=1}^{M}$ in (34). Moreover, any TRS with equal Voronoi regions is generated by a group-covariant set of unitary matrices.

A natural question is whether other TRSs are weighted 1-designs with weights dependent on the areas of Voronoi regions. We now present an example 
to demonstrate that it can also hold for other classes of TRSs. Consider the frame obtained from quadric Alltop construction generated from cyclic shifts of the following four vectors [1]:

$$
\begin{aligned}
& \mathbf{a}_{1}=\frac{1}{\sqrt{5}}\left(1, \omega_{5}, \omega_{5}^{4}, \omega_{5}^{4}, \omega_{5}\right), \\
& \mathbf{a}_{2}=\frac{1}{\sqrt{5}}\left(1, \omega_{5}^{2}, \omega_{5}^{3}, \omega_{5}^{3}, \omega_{5}^{2}\right), \\
& \mathbf{a}_{3}=\frac{1}{\sqrt{5}}\left(1, \omega_{5}^{3}, \omega_{5}^{2}, \omega_{5}^{2}, \omega_{5}^{3}\right), \\
& \mathbf{a}_{4}=\frac{1}{\sqrt{5}}\left(1, \omega_{5}^{4}, \omega_{5}, \omega_{5}, \omega_{5}^{4}\right),
\end{aligned}
$$

where $\omega_{5}=\exp (2 \pi i / 5)$. This collection of vectors is known to be equivalent to a set of MUBs [1, Theorem 1]. We have already seen that MUBs form complex projective 2-designs (see Proposition 3) and now seek to understand when they are also weighted 1-designs with non-uniform weights given by the areas of Voronoi regions of the frame elements.

To this end, observe that the elements can all be generated by applying the following unitary transformations.

$$
\begin{aligned}
\mathbf{U}_{1}=\left(\begin{array}{ccccc}
1 & 0 & 0 & 0 & 0 \\
0 & \omega_{5} & 0 & 0 & 0 \\
0 & 0 & \omega_{5}^{4} & 0 & 0 \\
0 & 0 & 0 & \omega_{5}^{4} & 0 \\
0 & 0 & 0 & 0 & \omega_{5}
\end{array}\right), & \mathbf{U}_{2}=\left(\begin{array}{ccccc}
1 & 0 & 0 & 0 & 0 \\
0 & \omega_{5}^{2} & 0 & 0 & 0 \\
0 & 0 & \omega_{5}^{3} & 0 & 0 \\
0 & 0 & 0 & \omega_{5}^{3} & 0 \\
0 & 0 & 0 & 0 & \omega_{5}^{2}
\end{array}\right), \\
\mathbf{U}_{3}=\left(\begin{array}{ccccc}
1 & 0 & 0 & 0 & 0 \\
0 & \omega_{5}^{3} & 0 & 0 & 0 \\
0 & 0 & \omega_{5}^{2} & 0 & 0 \\
0 & 0 & 0 & \omega_{5}^{2} & 0 \\
0 & 0 & 0 & 0 & \omega_{5}^{3}
\end{array}\right), & \mathbf{U}_{4}=\left(\begin{array}{ccccc}
1 & 0 & 0 & 0 & 0 \\
0 & \omega_{5}^{4} & 0 & 0 & 0 \\
0 & 0 & \omega_{5} & 0 & 0 \\
0 & 0 & 0 & \omega_{5} & 0 \\
0 & 0 & 0 & 0 & \omega_{5}^{4}
\end{array}\right) .
\end{aligned}
$$

The frame obtained from (38) is then obtained from $\left\{\sigma_{j} \mathbf{U}_{i} \frac{1}{\sqrt{5}} \mathbf{1}\right\}_{j, i}$, where $\left\{\sigma_{j}\right\}$ is the unitary representation of the group of cyclic shifts on 5 letters. It then follows that

$$
\mathbf{U}_{1}=\sigma \mathbf{U}_{4}
$$

and

$$
\mathbf{U}_{2}=\sigma^{\prime} \mathbf{U}_{3},
$$

where $\sigma, \sigma^{\prime}$ are elements of the unitary representation for the group of permutations on 5 letters. 
Now observe that

$$
\begin{aligned}
V_{i} & =\mu\left(\left\{\left.\mathbf{z} \in S^{d-1}|| \mathbf{z}^{\dagger} \boldsymbol{\varphi}_{i}\right|^{2} \geq\left|\mathbf{z}^{\dagger} \boldsymbol{\varphi}_{j}\right|^{2}, j \neq i\right\}\right) \\
& =\mu\left(\left\{\left.\mathbf{z} \in S^{d-1}|| \mathbf{z}^{\dagger} \sigma \boldsymbol{\varphi}_{i}\right|^{2} \geq\left|\mathbf{z}^{\dagger} \boldsymbol{\varphi}_{j}\right|^{2}, j \neq i\right\}\right),
\end{aligned}
$$

which means that the permutations of $\boldsymbol{\varphi}_{i}$ (obtained from $\mathbf{U}_{i} \frac{1}{\sqrt{5}} \mathbf{1}$ ) all have the same Voronoi areas. Moreover, since (41) and (42) hold, it follows that $\boldsymbol{\varphi}_{1}$ and $\varphi_{4}$ (and all their cyclic shifts) have the same Voronoi areas. Similarly $\varphi_{2}$ and $\varphi_{3}$ have the same Voronoi areas.

To show that equality is achieved in (36), observe that

$$
\begin{aligned}
\sum_{i} \sum_{j}\left|\boldsymbol{\varphi}_{i}^{\dagger} \boldsymbol{\varphi}_{j}\right|^{2} V_{i} V_{j} & =10 \alpha^{2}+10 \beta^{2}+50 \alpha^{2} / 5+50 \beta^{2} / 5+200 \alpha \beta / 5 \\
& =20\left(\alpha^{2}+\beta^{2}\right)+40 \alpha \beta
\end{aligned}
$$

where $\alpha, \beta$ are the Voronoi areas. Furthermore, $10 \alpha+10 \beta=1$, which means that

$$
\sum_{i} \sum_{j}\left|\boldsymbol{\varphi}_{i}^{\dagger} \boldsymbol{\varphi}_{j}\right|^{2} V_{i} V_{j}=20 / 100=1 / 5=1 / d
$$

as required.

We remark that a large class of TRSs appear to be weighted 1-design with weights given by the areas of frame element Voronoi regions. To illustrate this, we have numerically estimated via Monte Carlo simulations the value

$$
Q=\sum_{i} \sum_{j}\left|\boldsymbol{\varphi}_{i}^{\dagger} \boldsymbol{\varphi}_{j}\right|^{2} V_{i} V_{j}
$$

for several constructions of ETFs and MUBs. A selection of the results are presented in Table 1. Observe that the results suggest that for each construction, the weighted 1-design property holds.

Table 1 Numerical Study of TRSs

\begin{tabular}{|c|c|c|}
\hline \hline Construction & Estimated $Q$ in $(46)$ & $\frac{1}{d}$ \\
\hline$(2,2,4)$-Steiner system ETF $[13$, Section 2$]$ & 0.1666 & $\frac{1}{6}$ \\
\hline$\{1,2,4\}$-Difference set ETF $[28]$ & 0.3333 & $\frac{1}{3}$ \\
\hline$\{0,3,5,6\}$-Difference set ETF $[28]$ & 0.25 & $\frac{1}{4}$ \\
\hline$\{0,1,3,9\}$-Difference set ETF $[28]$ & 0.25 & $\frac{1}{4}$ \\
\hline$\{1,3,4,5,9\}$-Difference set ETF $[28]$ & 0.2 & $\frac{1}{5}$ \\
\hline$\{3,6,7,12,14\}$-Difference set ETF $[28]$ & 0.2 & $\frac{1}{5}$ \\
\hline$\{0,2,6,7,8,10\}$-Difference set ETF $[28]$ & 0.1667 & $\frac{1}{6}$ \\
\hline$\{1,5,11,24,25,27\}$-Difference set ETF $[28]$ & 0.1667 & $\frac{1}{6}$ \\
\hline$\{0,1,2,4,5,8,10\}$-Difference set ETF $[28]$ & 0.1429 & $\frac{1}{7}$ \\
\hline Alltop Quadric MUB $N=7[1]$ & 0.1429 & $\frac{1}{7}$ \\
\hline Alltop Cubic MUB $p=5[1]$ & 0.2000 & $\frac{1}{5}$ \\
\hline
\end{tabular}


We also remark that these numerical results complement Theorem 5, which shows that all TRSs constructed via group covariant sets are such weighted 1-designs. Moreover, the maximal ETFs corresponding to the case $M=d^{2}$ in [20] are obtained through the action of the Weyl-Heisenberg group and hence by Theorem 5 , they are also weighted 1-designs with weights determined by the Voronoi regions.

These observations motivate the following conjecture linking TRSs and weighted 1-designs.

Conjecture $1 \mathrm{In}\left(\boldsymbol{\varphi}_{i}\right)_{i=1}^{M}$ in $\mathbb{C}^{d}$ is a TRS, then it is also a weighted 1-design with weights given by the areas of the Voronoi regions $V_{i}$ in (34) for each frame element.

\section{References}

1. Alltop, W.: Complex sequences with low periodic correlations. IEEE Transactions on Information Theory 26(3), 350-354 (1980)

2. Benedetto, J., Fickus, M.: Finite normalized tight frames. Advances in Computational Mathematics 18(2-4), 357-385 (2003)

3. Calderbank, A., Cameron, P., Kantor, W., Seidel, J.: $\mathbb{Z}_{4}$-Kerdock codes, orthogona spreads, and extremal Euclidean line-sets. Proc. London Math. Soc. 75(3), 436-480 (1997)

4. Casazza, P., Kutyniok, G.: Finite Frames: Theory and applications. Springer Science and Business Media (2012)

5. Chang, G.: Complete diagonals of Latin squares. Canadian Journal of Mathematics 22(4), 477-481 (1979)

6. Datta, S., Oldroyd, J.: Construction of $k$-angle tight frames. Numerical Functional Analysis and Optimization 37(8), 975-989 (2016)

7. Delsarte, P., Goethals, J., Seidel, J.: Bounds for systems of lines and Jacobi polynomials. Philips Res. Rep. 30, 91-105 (1975)

8. Ding, C., Feng, T.: A generic construction of complex codebooks meeting the Welch bound. IEEE Transactions on Information Theory 53(11), 4245-4250 (2007)

9. Ding, C., Mesnager, S., Tang, C., Xiong, M.: Cyclic bent functions and their applications in codes, codebooks, designs, MUBs and sequences. arXiv:1811.07725 (2018)

10. Ding, C., Yin, J.: Signal sets from functions with optimal nonlinearity. IEEE Transactions on Communications 55(5), 936-940 (2007)

11. Durt, T., Englert, B.G., Bentsson, I., Zyczkowski, K.: On mutually unbiased bases. International Journal of Quantum Information 8, 535-640 (2010)

12. Egan, M., Sung, C., Collings, I.: Structured and sparse limited feedback codebooks for multiuser MIMO. IEEE Transactions on Wireless Communications 12(8), 3710-3721 (2013)

13. Fickus, M., Mixon, D., Tremain, T.: Steiner equiangular tight frames. Linear Algebra Appl. 436(5)

14. Haas, J., Hammen, N., Mixon, D.: The levenshtein bound for packings in projective spaces. In: Wavelets and Sparsity XVII (2017)

15. Heng, Z., Yue, Q.: Optimal codebooks achieving the Levenshtein bound from generalized bent functions over $\mathbb{Z}_{4}$. Cryptogr. Commun. 9, 41-53 (2017)

16. Hoggar, S.: $t$-Designs in projective spaces. Europ. J. Combinatorics 3, 233-254 (1982)

17. Hoggar, S.: Parameters of $t$-designs in $\mathbb{F} P^{d-1}$. Europ. J. Combinatorics 5, 29-36 (1984)

18. Klappenecker, A., Rotteler, M.: Mutually unbiased bases are complex projective 2designs. In: IEEE International Symposium on Information Theory (2005)

19. Levenstein, V.: Bounds for packing of metric spaces and some of their applications. Probl. Cybern. 40, 43-110 (1983) 
20. Renes, J., Blume-Kohout, R., Scott, A., Caves, C.: Symmetric informationally complete quantum measurements. Journal of Mathematical Physics 45(6), 2171-2180 (2004)

21. Roy, A., Scott, A.: Weighted complex projective 2-designs from bases: optimal state determination by orthogonal measurements. Journal of Mathematical Physics 48(7) (2007)

22. Strohmer, T., Heath Jr., R.: Grassmannian frames with applications to coding and communication. Applied and Computational Harmonic Analysis 14(3), 257-275 (2003)

23. Thill, M., Hassibi, B.: Group frames with few distinct inner products and low coherence. IEEE Transactions on Signal Processing 63(19), 5222-5237 (2015)

24. Vale, R., Waldron, S.: Tight frames and their symmetries. Constructive Approximation 21(1), 83-112 (2004)

25. Waldron, S.: An Introduction to Finite Tight Frames. Birkhäuser (2018)

26. Welch, L.: Lower bounds on the maximum cross-correlation of signals. IEEE Transactions on Information Theory 20(3), 397-399 (1974)

27. Wootters, W., Fields, B.: Optimal state-determination by mutually unbiased measurements. Annals of Physics 191(2), 363-381 (1989)

28. Xia, P., Zhou, S., Giannakis, G.: Achieving the Welch bound with difference sets. IEEE Transactions on Information Theory 51(5), 1900-1907 (2005)

29. Xiang, C., Ding, C., Mesnager, S.: Optimal codebooks from binary codes meeting the Levenshtein bound. IEEE Transactions on Information Theory 61(12), 6526-6535 (2015)

30. Zhou, Z., Ding, C., Li, N.: New families of codebooks achieving the Levenshtein bound. IEEE Transactions on Information Theory 60(11), 7382-7387 (2014) 